\title{
Ganglioglioma of Conus Medullaris-A Rare Case
}

\author{
Sushil Kumar ${ }^{1}$ Rajneesh Misra ${ }^{1} \quad$ Kundan Kumar $^{1}$ Sandeep Sharma ${ }^{1}$ \\ ${ }^{1}$ Department of Neurosurgery, St. Stephen's Hospital, Tis Hazari, \\ Delhi, India \\ Address for correspondence Rajneesh Misra, MS, Mch, \\ Department of Neurosurgery, St. Stephen's Hospital, Tis Hazari, \\ Delhi, 110054, India (e-mail: misra_rajneesh@yahoo.com).
}

\begin{abstract}
Gangliogliomas favor the temporal lobe. They are rarely reported in the spinal cord. Ganglioglioma of the conus medullaris is very rare. An 11-year-old boy presented with progressive weakness of bilateral lower limbs. Clinical examination and radiologic investigations revealed a lesion in the conus medullaris with an exophytic component involving the cauda equina roots. The lesion was excised near totally. Its histopatho-

Keywords

- ganglioglioma

- intramedullary

- exophytic

- conus medullaris logic examination revealed it to be a ganglioglioma. Gangliogliomas of the conus medullaris are rare lesions. Subtotal or near-total excision with preservation of the function should be the aim of the surgical intervention. Because preoperative function largely dictates the postoperative course, it is advisable to intervene early at the first hint of neurologic compromise.
\end{abstract}

\section{Introduction}

Gangliogliomas are benign slow-growing tumors of the central nervous system, most often affecting the temporal lobe. Their location in the spinal cord is rare..$^{1-4}$ Usually they are intramedullary, and exophytic extension is very rare. It is because of this rare location that this case is being reported.

\section{Case Report}

An 11-year-old boy was admitted with complaints of pain, weakness of the right lower limb, and difficulty in walking of 1 -year duration.On examination, tone in the lower limbs was normal. Power was Gr-V at the hip and knee and Gr-III at the ankle and toes on the right side. It was Gr-V in all groups of muscles in the left lower limb. All modalities of sensations were normal. There was no spinal deformity or tenderness. Magnetic resonance imaging (MRI) of the lumbar spine showed expansive mass lesion extending from D11 to L2 vertebra. It was isointense on plain T1W1 and hyperintense on T2W1. It showed inhomogeneous contrast enhancement and

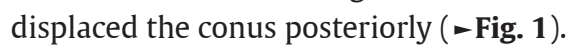

Laminectomy of D11 and laminoplasty of D12-L2 revealed expanded cord with fleshy, grayish, pinkish, moderately vascular tumor arising from right anterolateral part of conus surrounding the roots of cauda equina up to L2 vertebra. Tumor was gradually separated from the roots, and near-total excision of tumor was done. Postoperatively, the patient developed
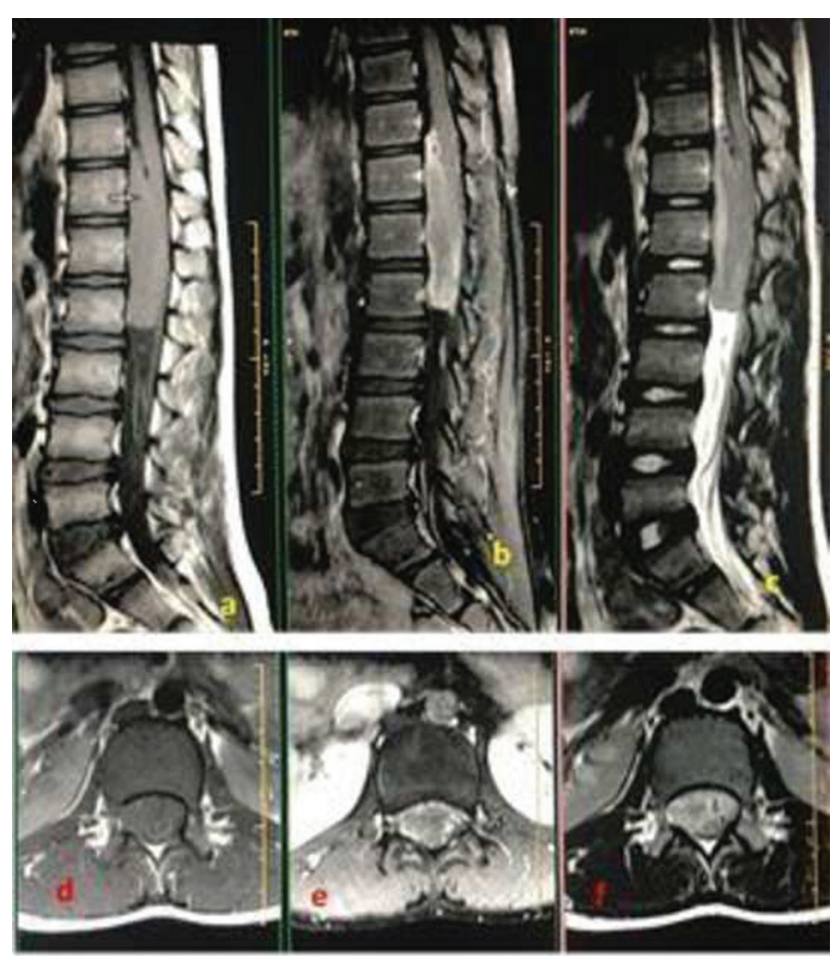

Fig. 1 Upper row: Sagittal MRI showing (a) isointense mass on TIWI with exophytic component extending from T11 to L2, (b) inhomogeneous contrast enhancement, (c) hyperintense signals anteriorly and posteriorly. Lower row: Axial MRI showing (d) isointense diffuse mass on TIWI, (e) inhomogeneous contrast enhancement, and (f) hyperintense mass pushing the cord posteriorly and to the left. received

February 22, 2019

accepted

May 1, 2019

published online

November 15, 2019
Dol https://doi.org/

10.1055/s-0039-1700301

ISSN 2277-954X.
C2019 Neurological Surgeons'

Society of India
License terms

()(1) $\Theta \circledast$ 
paraplegia, within 1 week power improved to Gr-III, and he was discharged with advice to continue physiotherapy exercises.

Histopathology revealed the tumor cells composed of mixture of neoplastic astrocytes displaying mild nuclear pleomorphism admixed with variable-sized dysplastic and multinucleated ganglion cells ( - Fig. 2 ). There was no evidence of necrosis or mitotic activity. Tumor cells were positive for synaptophysin, glial fibrillary acidic protein (GFAP), and focally for CD34. Histopathology and immunochemistry confirmed the diagnosis of ganglioglioma (Gr-I).

\section{Discussion}

Spinal gangliogliomas are more frequent in cervicothoracic region, with the conus medullaris being an extremely uncommon site. Sunder et al reported a case of conus ganglioglioma who had associated neurofibromatosis type $1 .^{5}$ Sandeep et al could find 15 reported cases of ganglioglioma of the conus medullaris and added one of their own. ${ }^{6}$

Usually the patients present with pain, paraparesis, gait ataxia, sensory disturbances, and bowel or bladder

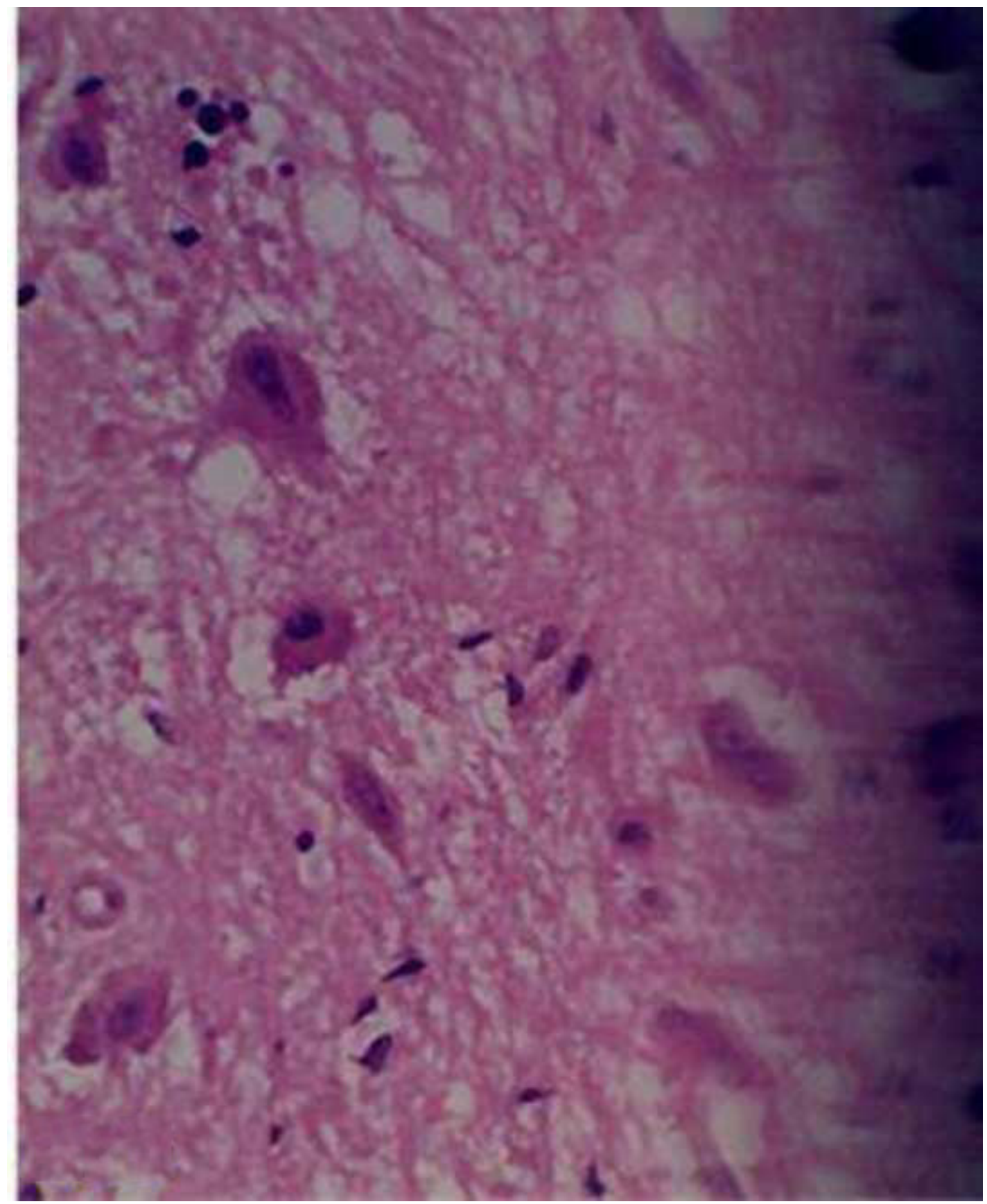

Fig. 2 Photomicrograph showing neoplastic astrocytes with variable-sized multinucleated ganglion cells (H\&E 200×). 
dysfunction. There are no pathognomonic radiologic features. MRI shows mixed intensities on TIWI and homogenous on T2WI and patchy contrast enhancement of tumor and cord surface. Most often, there is no perilesional edema. Gross total surgical resection is the treatment of choice if there is good plane of cleavage; however, complete excision may not be feasible if plane of cleavage is not there.

In our case exophytic part was removed easily, and intratumoral gross removal of intramedullary portion was done. Recurrence rate is low and requires redo surgery. There is no role of chemotherapy as the lesion is benign in nature. Malignant transformation is extremely rare. ${ }^{3}$

Preoperative function is predictor of prognosis, so early operative intervention results in better outcome. Tumors are positive for synaptophysin, neurofilament protein, neurospecific enolase, and chromogranin A, and glial element expresses GFAP on immunohistochemistry.

To conclude, ganglioglioma of the conus medullaris are rare lesions. Subtotal or near-total excision with preservation of the function should be the aim of the surgical intervention. Because preoperative function largely dictates the postoperative course, it is advisable to intervene early at the first hint of neurologic compromise.

\section{Conflicts of Interest}

The authors declare no conflict of interest.

\section{References}

1 Nass R, Whelan MA. Gangliogliomas. Neuroradiology 1981;22(2):67-71

2 Costa J, Ruivo J, Miguéns J, Pimentel J. Ganglioglioma of conus medullaris. Acta Neurochir (Wien) 2006;148(9):977-980

3 Amini A, Chin SS, Schmidt MH. Malignant transformation of conus medullaris ganglioglioma: case report. J Neurooncol 2007;82(3):313-315

4 Hicdonmez T, Suslu HT, Yavuzer D, Tatarli N. Paediatric ganglioglioma of the conus medullaris. J Clin Neurosci 2011;18(8):1124-1126

5 Sundar IV, Jaiswal M, Purohit D, Mittal RS. Ganglioglioma of conus medullaris in a patient of neurofibromatosis type 1: a novel association? Asian J Neurosurg 2016;11(4):455-457

6 Sandeep BV, Roy K, Saha SK, Banga MS. Intramedullary spinal ganglioglioma involving the conus with unusual magnetic resonance imaging features. J Spinal Surg 2016;3:114-116 